\title{
Precise and Automatic Anthropometric Measurement Extraction Using Template Registration
}

\author{
Oliver WASENMÜLLER, Jan C. PETERS, Vladislav GOLYANIK, Didier STRICKER \\ German Research Center for Artificial Intelligence (DFKI), Kaiserslautern, Germany
}

\author{
DOI: 10.15221/15.155 http://dx.doi.org/10.15221/15.155
}

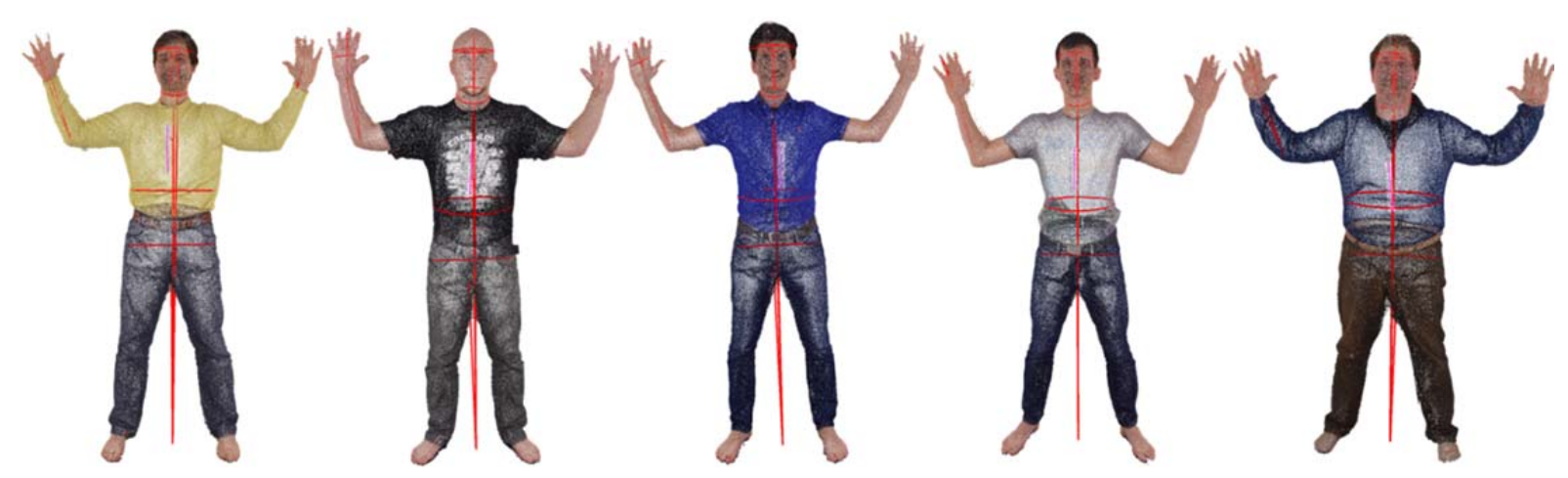

Fig. 1. Extracted anthropometric measurements for five exemplary human body scans.

\begin{abstract}
Anthropometric measures build the basis for many applications, such as custom clothing or biometric identity verification. Consequentially, the possibility to automatically extract them from human body scans is of high importance. In this paper we present a new approach based on landmarks and template registration. First, we propose a new method to define anthropometric measures once on a generic template using landmarks. After the initial definition the template can be registered against an individual body scan and the landmarks can be transferred to the scan using our second proposed algorithm. We apply our complete approach to real and synthetic human data and show that it outperforms the state-of-the-art for several measures.
\end{abstract}

Keywords: anthropometry, measurement extraction, landmark definition, template registration

\section{Introduction}

Nowadays several approaches, ranking from low cost scanners [5] to professional scanners [7], exist to reconstruct a precise 3D model of a human body. To use these models in applications such as custom clothing, biometric identity verification and virtual try-on, approaches to extract anthropometric data from human body scans are required. The extracted measures build the basis for further processing and thus automatic and reliable approaches are of importance. The literature proposes semi-automatic and automatic approaches like detecting landmarks [10] or using prior knowledge by regression learning [13], each with particular drawbacks.

Our new approach follows the idea to extract anthropometric measurements according to landmarks and also incorporates prior knowledge. When relying on these landmarks, their precise localization becomes a crucial point. Besides manual positioning, the literature proposes to place them automatically according to a skeleton [10] or body segmentation [9]. However, in both cases landmarks are still too coarse when targeting at precise results in real-world applications. Thus, we propose within this paper the following new approach. We define the landmarks on a generic template once and align and morph the template to individual human body scan by a non-rigid registration algorithm. The landmarks are transferred from the registered template to the scan and consequently individual anthropometric measures can be extracted. Hence, this is a hybrid approach that uses landmarks as well as prior knowledge in terms of measurement definitions on a generic template.

For evaluation we used real and synthetic body scans. As shown in Fig. 1, the anthropometric measures are placed precisely on the human bodies. To estimate the precision of our approach we also used synthetic scans with the advantage that ground truth reference data is provided.

*oliver.wasenmueller@dfki.de 
The paper is organized as follows: In Section 2 we present related work, while in Section 3 we propose our new method for automatic anthropometric measurement extraction. We evaluate our method in Section 4 and conclude in Section 5.

\section{Related Work}

In the literature several approaches for anthropometric measurement extraction are described. In principle they can be subdivided in two main categories: approaches based on prior knowledge and approaches based on landmarks.

Approaches based on prior knowledge use human models with known measures against which comparison is undertaken. Buys et al. [3] proposed a simple approach, where they create human-like meshes with MakeHuman [11] with known anthropometric measures, render them and compare thresholded versions of the rendered images and real images of the respective person. The parameters of MakeHuman are adjusted until the best image correspondences are achieved. Tsoli et al. [13] and Weiss et al. [15] proposed more advanced approaches, where they use regression learning to transfer the given human body model into variants of the Shape Completion and Animation of People (SCAPE) [1] representation. SCAPE is a mathematical model describing pose and body shape deformations. After transferring a given body scan into this model one can directly derive anthropometric measures. All presented approaches, which are based on prior knowledge, have the disadvantage of not being able capable to estimate directly on a given scan, but derive measures out of a mathematical model.

The second category covers approaches based on so-called landmarks, which are characteristic points on the scan surface defining anthropometric measures. The crucial point is how these landmarks are positioned on individual body scans. Lovato et al. [10] proposed a combined method which extracts a skeleton out of the body scan to use the joints as landmarks, but also provides a graphical interface for semi-automatic measurement extraction. This results in a precise, but still not fully automatic technique for anthropometric measurement extraction. Li and Wang [9] proposed an approach, where they segment the human body according to its extremities and use the transitions as landmarks. However, all these landmark based approaches in common suffer from their imprecise positioning of the landmarks.

Thus, we propose in this paper a hybrid approach using a generic template with measurement definitions as prior knowledge and use them to position landmarks on individual human body scans. In such a way we try to overcome the particular disadvantages of previous methods and enable robust landmark positioning. Besides comparing our approach to the state-of-the-art we also consider the ANSUR allowable error [6], which is the expected accuracy of manual measurements.

\section{Proposed Approach}

Our algorithm assumes a generic template, where anthropometric measures are defined once with the help of landmarks (see Section 3.1). This template is aligned and morphed to the human body scan, which should be anthropometrically analyzed, by a non-rigid registration algorithm (see Section 3.2). The landmarks are then transferred from the template to the scan and enhanced in their precision, if required (see Section 3.3). In the following sections our measurement extraction algorithm is described in more detail, whereas Fig. 2 gives an overview.

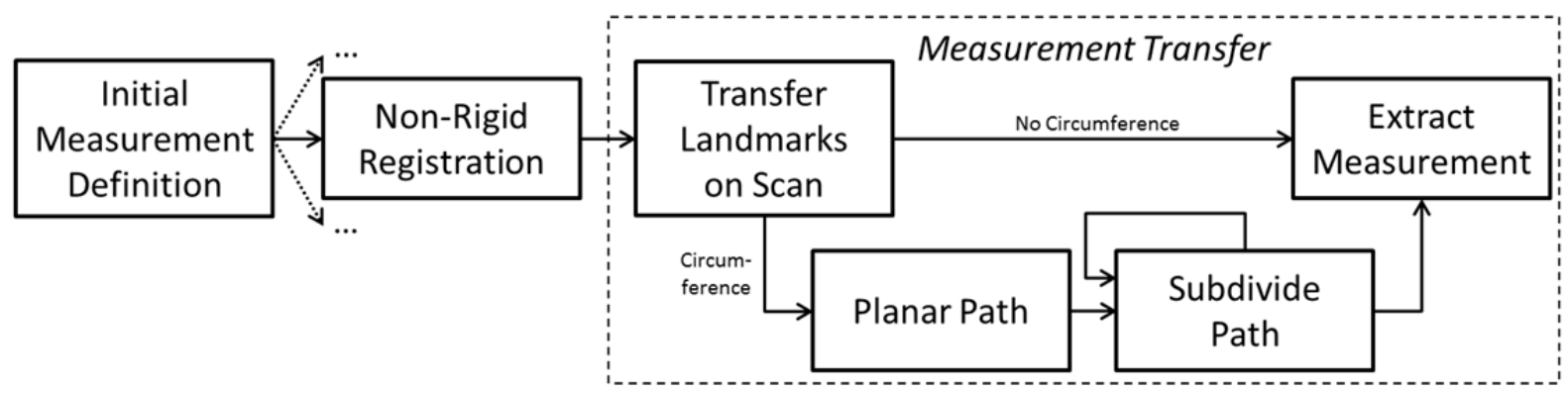

Fig. 2. Overview of our proposed approach. Measurements are initially defined on a generic template using landmarks. This template can be registered to individual body scans. Landmarks are transferred to the scan and measurements are extracted. In case of circumferences paths are subdivided and made planar. 


\subsection{Measurement Definition}

For our approach an anthropometric measure is defined as the length of its corresponding measurement path. A measurement path is defined by consecutive landmarks (see Fig. 3 left) and its length is the sum of Euclidean distances between these landmarks. A landmark is a characteristic point on the human body.

Simple anthropometric measures are given by at least two landmarks representing the start and end point of the measurement path. For instance, the lower arm length, which is defined by the landmarks 'wrist' and 'elbow'. Measures like the complete arm length are defined by the three landmarks 'wrist', 'elbow' and 'shoulder joint'. Circumference measurements are defined by a closed path having the same start and end landmark. The abdominal girth is e.g. described by several landmarks forming a ring on the waist.

In rare cases measures cannot be defined by landmarks on the scan surface. Thus, we define some landmarks implicitly as the average of selected points on the surface. For instance, the body height is estimated by the distance between an explicit landmark on the top of the cranium and an implicit landmark defined by four landmarks on the respective heels and balls of the feet as visible in Fig. 3 right. With all these definitions we try to realize the international standard ISO 7250.

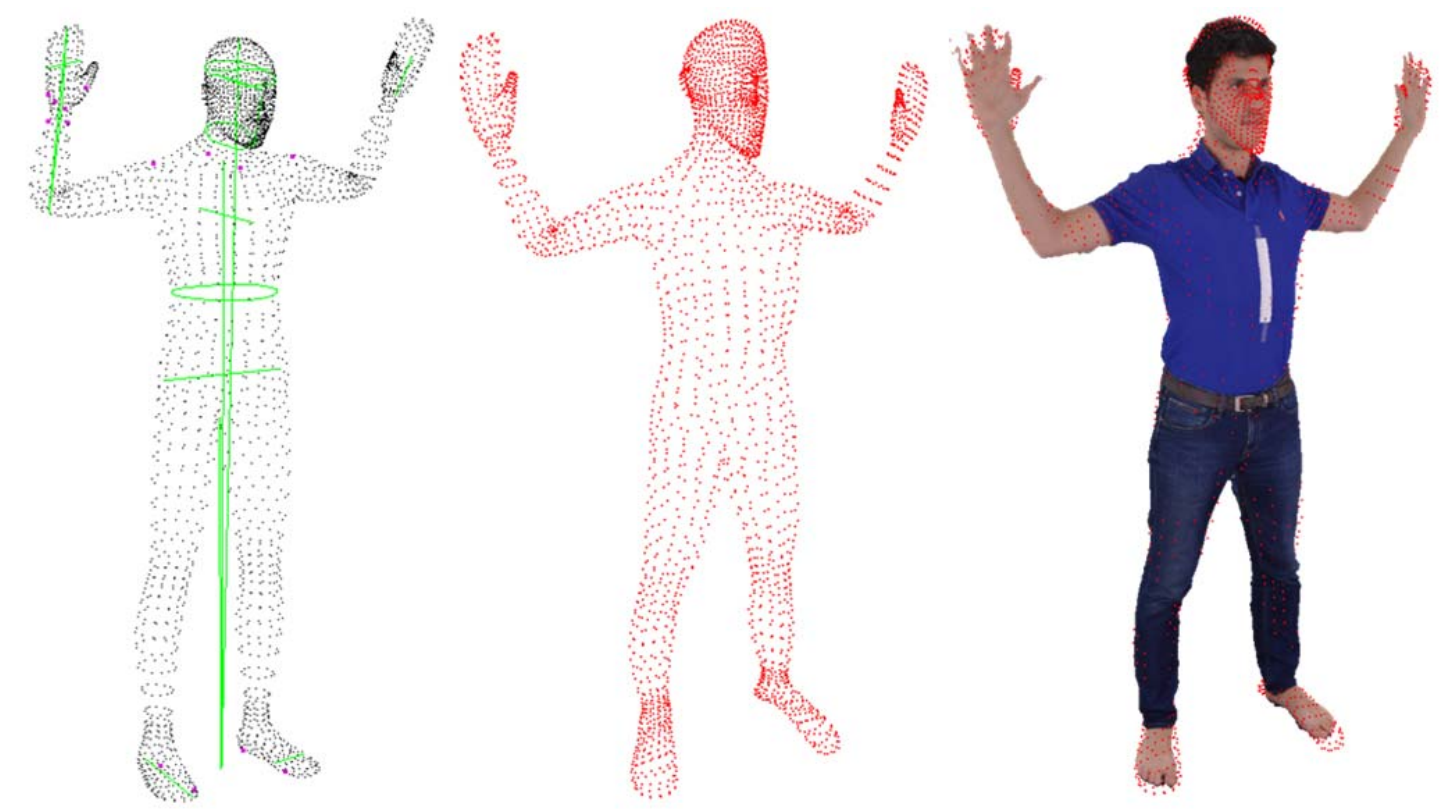

Fig. 3 Left: Generic template point cloud with some exemplary measurement paths. Measurement paths are marked in green; points defining indirect landmarks are emphasized with magenta (e.g. center between feet). Middle: Exemplary registered generic template point cloud. Right: Overlay of the registered template with the human body scan.

\subsection{Non-Rigid Registration}

To extract individual anthropometric measurements we align and morph the generic human body template with predefined measurement paths to the human body scan. Specifically, we take advantage of non-rigid point cloud registration, since it allows for general non-rigid transformations; in opposite to rigid registrations where transformation are defined by an identical rotation, translation and scale of all points. Thus, we obtain a scan-template registration (see Fig. 3), whereby initially steady shaped template body parts are aligned with variously shaped scan body parts. Consequently, the generally defined measurement paths on the registered template alter to individual measurement paths, for their part enabling extraction of individual anthropometric measurements.

One of the widely used non-rigid point cloud registration algorithms is non-rigid ICP [2]. It attempts to minimize the sum of square distances from every template's point to the respectively closest scan's point. Its main advantages are simplicity and fine registration results when point clouds are sufficiently similar and well pre-aligned. Nevertheless, our choice fell on the Gaussian Mixture Model (GMM) based Coherent Point Drift (CPD) [12]. Compared to non-rigid ICP, its performance is more stable in presence of noise and outliers. Since real-world scans are noisy point clouds, this property is determining for our application. Though several variants of Mixture Model based point cloud registration algorithms exist [8][16] including extensions of CPD [14], none of them can be considered as notably superior to CPD. 
CPD interprets point cloud registration as a probability density estimation problem. Positions of the template points determine centroids of the Gaussians combined into a GMM. The optimization objective is to find the GMM which explains the reference point cloud with the highest probability. For further information on CPD please refer to the original article [12]. Note that we initially pre-align the generic template and the body scan with the rigid version of CPD and subsequently proceed with the non-rigid template-scan registration. The quality of the CPD can be slightly increased when using additional constraints for the registration.

\subsection{Measurement Transfer}

Since small gaps between registered template and scan are unavoidable (see Fig. 3 right), we transfer the landmarks onto the scan. The crucial point is how to find for each landmark the corresponding point on the scan. An easy approach would be to look for each landmark for the closest point on the scan. However, this causes wrong assignments in concave regions like the armpit. This problem can be avoided by also considering the normal direction. Thus, we detect for each landmark $l$ points $x_{i}$ on the scan with short distance and similar normal direction $\overrightarrow{n_{l}}$ by maximizing

$$
\mathrm{l}^{\prime}=\arg \max _{x_{i}} \frac{\left|\overrightarrow{\left(l-x_{l}\right)} \cdot \overrightarrow{n_{l}}\right|}{\left\|l-x_{i}\right\|}
$$

The transferred landmarks $l^{\prime}$ define the measurement paths allowing to determine individual anthropometric measures of the scan. While this works well for the implicit landmarks, measurement definitions describing circumferences suffer from a slightly jagged and body piercing path (see Fig. 4 left). Thus, we enhance the position of the transferred landmarks $l^{\prime}$ in order to create a planar path, which closely follows the scan surface, with the following two-step approach.

First, we compute a regression plane from all landmarks in the path. Then, we project all landmarks onto this plane and look for each projection for close vertices in the scan surface. To avoid body piercing paths we apply the curve subdivision scheme of Chaikin [4]. Therefore, we subdivide each sub-path, defined by the landmarks $l_{i}^{\prime}$ and $l_{i+1}^{\prime}$, at $1 / 4$ and $3 / 4$ of the line $\overline{l^{\prime}{ }_{i} l^{\prime}{ }_{i+1}}$. After each subdivision step we retransfer the new landmarks $l^{\prime \prime}$ back onto the point cloud to force the path onto the scan surface. The described two-step approach can be applied iteratively to increase the quality of results. With each iteration the number of landmarks is doubled.
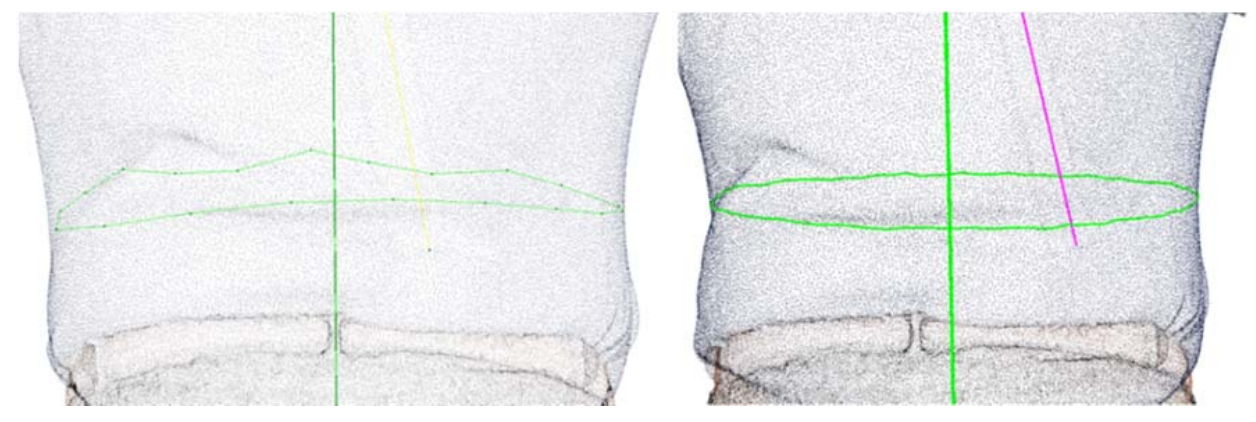

Fig. 4 Left: Original transferred hip circumference measurement path. Right: Enhanced hip circumference measurement path.

In the next step the transferred and corrected paths are evaluated by summing the Euclidean distances between subsequent landmarks $l^{\prime \prime}$. If the path contains indirect landmarks, their members are averaged and the resulting mean vertex is used to compute the distances.

\section{Evaluation}

In this Section we evaluate our algorithm. Therefore, we use two types of data. First, we apply our approach to real human body reconstructions showing its functionality. Since we have no ground truth reference for this data, we also applied our approach to synthetic data with known anthropometric measures in order to give quantitative conclusions.

For the evaluation with real world data we reconstructed bodies of several persons with a system similar to [7]. To estimate anthropometric measures, we used the generic template shown in Fig. 3 left and registered it against the body reconstructions. The results in Fig. 1 show that the paths for the anthropometric measures are placed precisely on the human bodies. The indirect landmarks between 
the feet for the measures Crotch Height and Body Height are positioned accurately. Also, the circumference measures like the Waist are placed correctly and form a perfect ring without any jitter. To estimate the precision of our approach we also used 30 synthetic scans, which were generated by MakeHuman [11] with the advantage that precise ground truth reference data is provided. As shown in Fig. 6 these models build a representative and comprehensive dataset with strongly varying body proportions.

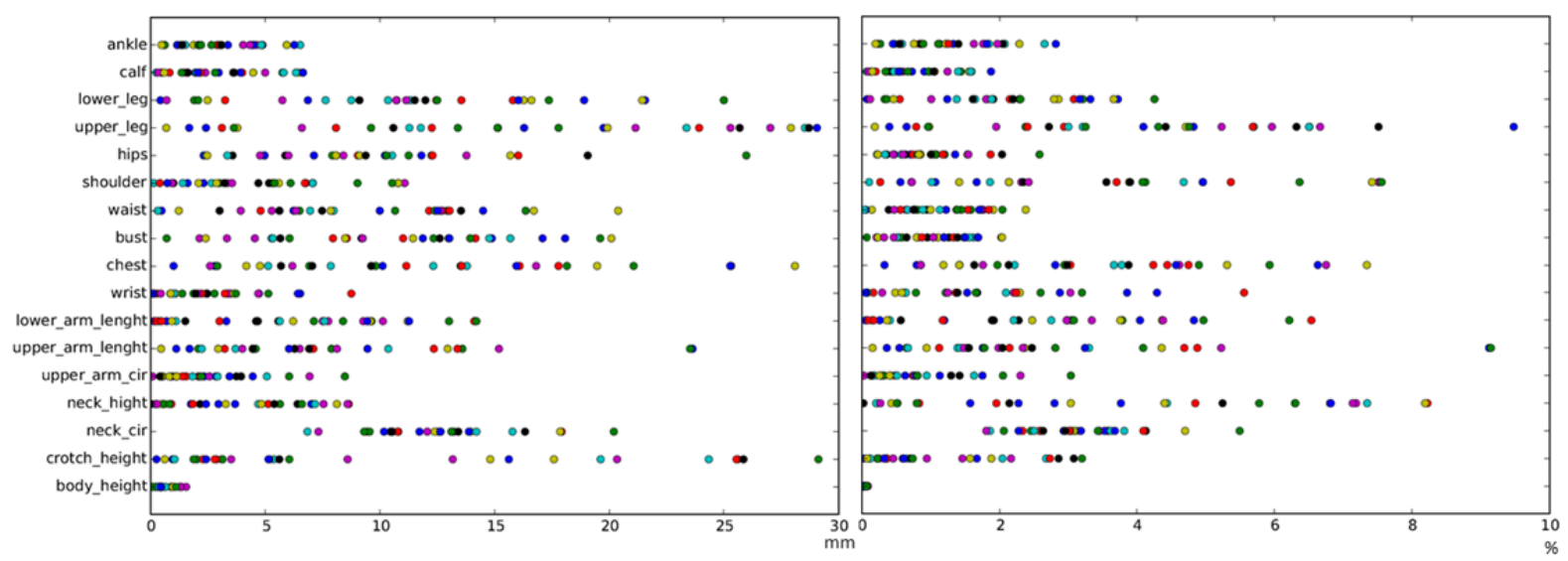

Fig. 5 Absolute deviation in millimeter (left) and relative deviation in percent (right) of the estimated anthropometric measures from the ground truth for the synthetic dataset.

Fig. 5 shows the absolute and relative deviations of our approach from the ground truth. For instance, we achieve errors of less than $7 \mathrm{~mm}$ for measures like Ankle, Calf or Body Height. The highest errors occur amongst others for the Upper Leg and Lower Leg, whereas the length of the complete leg is estimated correctly. The simple explanation is that it is difficult to place the landmark for the Knee precisely. In the non-rigid registration step a displacement of template points in top-down direction has a limited influence on the registration results in terms of cloud-to-cloud distance. However, it can increase the error in estimation of anthropometric measurements, since landmarks drift in this case.

We also compared a representative selection of our results against the state-of-the-art in Tab. 1. For measures like Ankle, Hip, Chest or Waist we outperform the competing approaches of Tsoli et al. [13] and Lovato et al. [10]. For measures like Upper Arm, Neck or Crotch competing approaches are slightly better. However, our results are at least in the range of the ANSUR Allowable Error (AE), which is the expected error of a manual measurement. Please note that the precision of the results also depends on the quality of the human body scans. This means, for other input data the results might negligibly vary.

\begin{tabular}{|l|r|r|r|r|}
\hline Measurement & ANSUR AE [6] & Tsoli et al. [13] & Lovato et al. [10] & Our Approach \\
\hline Ankle Circumference & 4.0 & 6.2 & $\sim 5.0$ & $\mathbf{3 . 1}$ \\
\hline Hip Circumference & 12.0 & 12.4 & - & $\mathbf{9 . 2}$ \\
\hline Chest Circumference & 15.0 & 12.7 & $\sim 23.0$ & $\mathbf{1 1 . 8}$ \\
\hline Waist Circumference & - & - & $\sim 19.0$ & $\mathbf{9 . 0}$ \\
\hline Upper Arm length & 6.0 & $\mathbf{6 . 7}$ & - & 7.7 \\
\hline Neck Circumference & 11.0 & 15.8 & $\sim 12.0$ & 12.4 \\
\hline Crotch Height & 10.0 & $\mathbf{5 . 5}$ & - & 9.8 \\
\hline
\end{tabular}

Tab. 1. Comparison of our results with the state-of-the-art. All values are given in millimeter. For several measures we outperform the competing approaches and achieve at least the similar accuracy as manual measuring (cp. ANSUR Allowable Error (AE)).

\section{Conclusion}

The main contribution of our paper is to use template registration to automatically extract anthropometric measurements from human body scans. Therefore, we proposed new approaches to define measurements on a generic template as well as an approach to transfer landmarks from a registered template to a body scan. We applied our complete method to real and synthetic data, showing a high precision. We outperform the state-of-the-art for several measures and achieve for all measures a similar accuracy as expected in manual measuring. Consequently, our approach is well suited to automatically extract anthropometric measures precisely. 


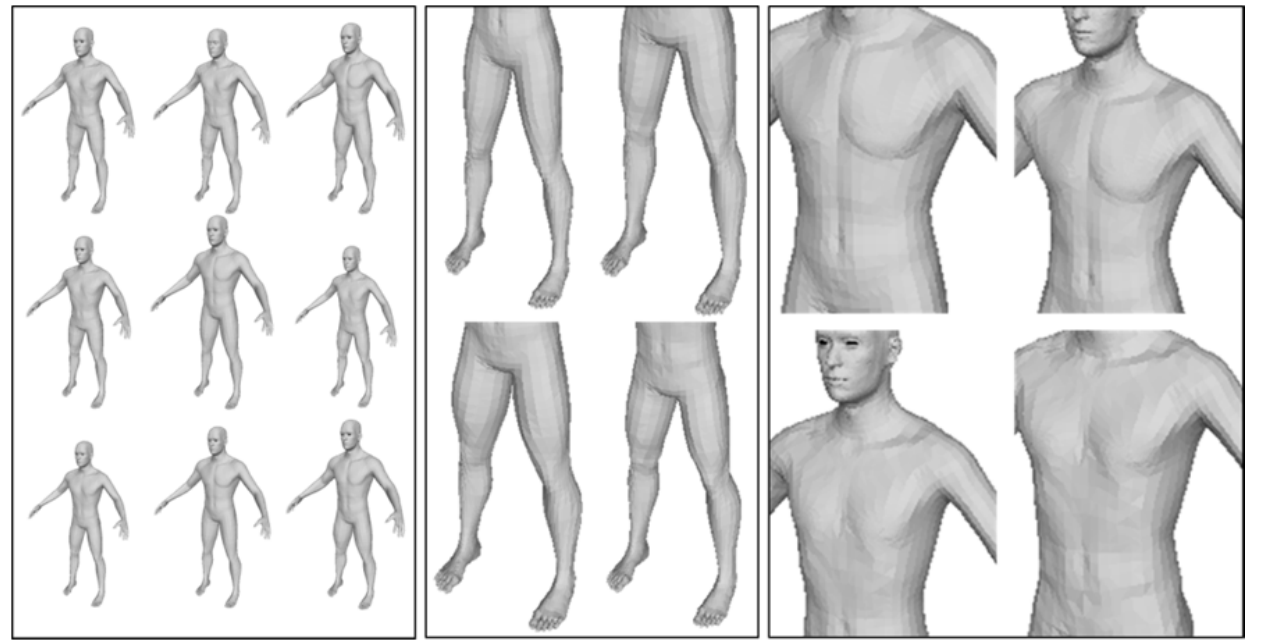

Fig. 6 Exemplary body models from the synthetic dataset with in total 30 different human bodies generated by MakeHuman [11]. Note especially the strongly varying body proportions within the dataset.

\section{Acknowledgments}

This work was partially funded by the Federal Ministry of Education and Research (Germany) in the context of the project Body Analyzer. We thank Michael Hödlmoser and Claudia Windisch from the Video Analytics Austria Researchgroup (CT RTC ICV VIA-AT) of Siemens for their support. Furthermore, we thank Bertram Taetz from the University of Kaiserslautern for the fruitful discussions.

\section{References}

[1] D. Anguelov et al. "SCAPE: Shape completion and animation of people", in ACM Transactions on Graphics (TOG), 2005, pp. 408-416.

[2] P.J. Besl and N.D. McKay. "A method for registration of 3-D shapes", in IEEE Transactions on Pattern Analysis and Machine Intelligence (PAMI), 1992, pp. 239-256.

[3] K. Buys et al. "Online generation of customized human models based on camera measurements". In International Symposium on Digital Human Modeling, 2011.

[4] G. M. Chaikin. "An algorithm for high-speed curve generation". In Computer Graphics and Image Processing, 1974, pp. 346-349.

[5] Y. Cui and D. Stricker, "3D Body Scanning With One Kinect", in Proc. of 2nd Int. Conf. on 3D Body Scanning Technologies, Lugano, Switzerland, 2011, pp. 121-129.

[6] C.C. Gordon et al. "Anthropometric survey of US army personnel: methods and summary statistics 1988", 1989.

[7] Infinite Realities, http://ir-ltd.net, accessed 2015.

[8] B. Jian and B.C. Vemuri. "Robust point set registration using Gaussian mixture models", in IEEE Transactions on Pattern Analysis and Machine Intelligence (PAMI), 2011, pp. 1633-1645.

[9] J. Li and Y.Wang. "Automatically constructing skeletons and parametric structures for polygonal human bodies", in Proc. of Computer Graphics International (CGI), Rio de Janeiro, Brazil, Vol. 7 , 2007, pp. 151-156.

[10]C. Lovato et al., "Computer assisted estimation of anthropometric parameters from whole body scanner data", in Modelling the Physiological Human, Lecture Notes in Computer Science, Vol. 5903, 2009, pp. 71-83.

[11]MakeHuman, www.makehuman.org, accessed 2015.

[12]A. Myronenko and S. Xubo. "Point Set Registration: Coherent Point Drift", in IEEE Transactions on Pattern Analysis and Machine Intelligence (PAMI), Vol. 32, No. 12 , 2010, pp. 2262-2275.

[13]A. Tsoli et al. "Model-based anthropometry: Predicting measurements from 3D human scans in multiple poses". In Proceedings Winter Conference on Applications of Computer Vision (WACV), 2014, pp. 83-90.

[14]P. Wang et al. "A refined coherent point drift (CPD) algorithm for point set registration", in Science China Information Sciences, 2011, pp. 2639-2646.

[15]A. Weiss et al. "Home 3D body scans from noisy image and range data". In IEEE International Conference on Computer Vision (ICCV), 2011, pp. 1951-1958.

[16]Z. Zhou et al. "Robust non-rigid point set registration using student's T mixture model", in PloS One, 2014. 